\title{
Team approach to polypharmacy evaluation and reduction: study protocol for a randomized controlled trial
}

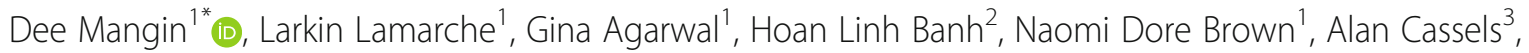
Kiska Colwill ${ }^{1}$, Lisa Dolovich ${ }^{1,4}$, Barbara Farrell ${ }^{5}$, Scott Garrison², James Gillett ${ }^{1}$, Lauren E. Griffith ${ }^{1}$, Anne Holbrook ${ }^{1}$, Jane Jurcic-Vrataric ${ }^{1}$, James McCormack ${ }^{6}$, Daria O'Reilly ${ }^{1}$, Parminder Raina', Julie Richardson ${ }^{1}$, Cathy Risdon', Mat Savelli ${ }^{1}$, Diana Sherifali ${ }^{1}$, Henry Siu${ }^{1}$, Jean-Éric Tarride ${ }^{1}$, Johanna Trimble ${ }^{6}$, Abbas Ali ${ }^{1}$, Karla Freeman $^{1}$, Jessica Langevin', Jenna Parascandalo', Jeffrey A. Templeton', Steven Dragos', Sayem Borhan ${ }^{1}$ and Lehana Thabane ${ }^{1}$

\begin{abstract}
Background: Polypharmacy in older adults can be associated with negative outcomes including falls, impaired cognition, reduced quality of life, and general and functional decline. It is not clear to what extent these are reversible if the number of medications is reduced. Primary care does not have a systematic approach for reducing inappropriate polypharmacy, and there are few, if any, approaches that account for the patient's priorities and preferences. The primary objective of this study is to test the effect of TAPER (Team Approach to Polypharmacy Evaluation and Reduction), a structured operationalized clinical pathway focused on reducing inappropriate polypharmacy. TAPER integrates evidence tools for identifying potentially inappropriate medications, tapering, and monitoring guidance and explicit elicitation of patient priorities and preferences. We aim to determine the effect of TAPER on the number of medications (primary outcome) and health-related outcomes associated with polypharmacy in older adults.

Methods: We designed a multi-center randomized controlled trial, with the lead implementation site in Hamilton, Ontario. Older adults aged 70 years or older who are on five or more medications will be eligible to participate. A total of 360 participants will be recruited. Participants will be assigned to either the control or intervention arm. The intervention involves a comprehensive multidisciplinary medication review by pharmacists and physicians in partnership with patients. This review will be focused on reducing medication burden, with the assumption that this will reduce the risks and harms of polypharmacy. The control group is a wait list, and control patients will be given appointments for the TAPER intervention at a date after the final outcome assessment. All patients will be followed up and outcomes measured in both groups at baseline and 6 months.
\end{abstract}

Discussion: Our trial is unique in its design in that it aims to introduce an operationalized structured clinical pathway aimed to reduce polypharmacy in a primary care setting while at the same time recording patient's goals and priorities for treatment.

\footnotetext{
* Correspondence: mangind@mcmaster.ca

'Department of Family Medicine, McMaster University, 100 Main Street West.,

5th floor, Hamilton, Ontario L8P 1H6, Canada

Full list of author information is available at the end of the article
}

\section{$\triangle B M C$}

C C The Author(s). 2021 Open Access This article is licensed under a Creative Commons Attribution 4.0 International License, which permits use, sharing, adaptation, distribution and reproduction in any medium or format, as long as you give appropriate credit to the original author(s) and the source, provide a link to the Creative Commons licence, and indicate if changes were made. The images or other third party material in this article are included in the article's Creative Commons licence, unless indicated otherwise in a credit line to the material. If material is not included in the article's Creative Commons licence and your intended use is not permitted by statutory regulation or exceeds the permitted use, you will need to obtain permission directly from the copyright holder. To view a copy of this licence, visit http://creativecommons.org/licenses/by/4.0/ The Creative Commons Public Domain Dedication waiver (http://creativecommons.org/publicdomain/zero/1.0/) applies to the data made available in this article, unless otherwise stated in a credit line to the data. 
Trial registration: Clinical Trials.gov NCT02942927. First registered on October 24, 2016.

Keywords: Polypharmacy, Overmedication, Multimorbidity, Deprescribing, Older adults, Medication reduction, Health outcomes, Operationalized clinical model, Physician pharmacist collaboration

\section{Background}

There is increasing concern about the harms of inappropriate medication use. Data suggest that adverse drug reactions may account for more morbidity and mortality annually than the potential benefit for the many chronic diseases the medications are designed to treat [1-5]. The death rates from adverse drug effects are higher than those from many common cancers $[4,6]$. There are particular concerns around polypharmacy in older adults. While there are varying definitions of polypharmacy, the most common definition is use of 5 or more long-term medications [7]. Polypharmacy is associated with negative health outcomes in older adults including falls, impaired cognition, and poorer nutrition [8-11]. Other potential negative associations include drug-drug interactions, drug-disease interactions, reduced medication adherence and difficulties in managing complicated medication regimes which exceed the patients' ability to cope [5, 10, 12-14].

The evidence for the effect of interventions to reduce polypharmacy on health outcomes shows inconsistent results, and difficulties in implementation; several reviews recommended further randomized controlled trials (RCTs) evaluating multidisciplinary interventions and clinical outcomes across different settings [10, 15-20]. Our feasibility study in 2010, a prospective cohort study of multiple medication discontinuation in communitydwelling adults, showed medication reduction and positive effects on health outcomes with no serious adverse effects [21]. Furthermore, a feasibility study in long-term care, targeting deprescribing of anticholinergic and sedative medicines, showed that medication reduction resulted in reductions in psychotropic drug side effects, falls, and depression and frailty scores [22]. Evidence shows that use of explicit lists of potentially inappropriate medicines (PIMs), such as the Screening Tool of Older Person's Prescriptions (STOPP) and Beers list criteria can improve appropriateness of prescribing in older adults [23-25]. As well, potential benefits of using explicit PIMs list for predicting adverse drug events have been found [26-30]. However, these studies provide insufficient evidence of the reversibility of the negative health effects associated with polypharmacy.

A number of proposed approaches to polypharmacy, broadly divisible into implicit and explicit approaches, have been reviewed in detail in a previous publication [10] and so they will not be discussed here. The tools available are largely used as decision aids to guide clinicians on which medicines are likely to be inappropriate in older patients or to cause adverse events, based on observational data and consensus [31]. More recently, electronic deprescribing aids that aggregate multiple sources of evidence have been developed. These and other screening tools have been developed from observational studies. These tools are typically explicit lists of individual PIMs, and indices of cumulative side effect burden which help to identify some problematic medications that are associated with hospitalizations due to adverse drug events, increased length of hospital stay, and increased risk and rate of falls [26-28, 32, 33]. Deprescribing guidelines have also been developed for some individual classes of medications, providing evidencebased decision algorithms as a pathway to reducing or stopping these medications in these classes under specific circumstances [34-38].

Explicit screening tools, while useful, are not sufficient as a complete approach to reducing harms of polypharmacy as they include by necessity only the most common, adverse drug events, or focus on selected adverse effects [39]. Further, such tools do not seek information directly from patients about side effects nor consider patientfocused prioritization [40]. Therefore, we carried out a systematic review of operationalized models recording patient preferences for medication use in the context of multimorbidity or polypharmacy [40]. We found no such models, though we did find a part of one tool that was valuable, as it assessed relative prioritization of symptom treatment versus treatments to prevent future illness, and has been validated in a similar population [41].

\section{Team Approach to Polypharmacy Evaluation and Reduction (TAPER)}

Primary care is strongly associated with improved health outcomes and is highlighted as an ideal setting for addressing polypharmacy: its generalist, comprehensive, person-focused approach to care, which offers integrated and longitudinal continuity [1]. However, there are no systematic approaches for family practice that are routinely embedded in clinical care for reducing inappropriate polypharmacy, and no interventions we are aware of that take a conceptual approach based on these core principles of primary care, and the core functions that support the effect of primary care on improved health outcomes (first contact care, comprehensiveness, continuity, person focus). A feasibility RCT of a Team Approach to Polypharmacy Evaluation and Reduction 
(TAPER) in a primary care setting in Canada similarly showed medication reduction and that the direction of most of the outcome measures supported the effectiveness of TAPER compared to usual care. This preliminary exploration supporting positive trends in effect across a variety of outcome measures [42] provide the support needed to proceed with this larger RCT powered to test the effects of TAPER, and to determine whether it can reduce medications numbers and affect health outcomes.

In this study, we propose to test TAPER, an intervention designed to be suitable for routine use in primary care. TAPER is seated within a theoretical framework of prevention (Quaternary Prevention, or "P4"), which includes actions designed to prevent the harms of unnecessary medication use, overtreatment, and overdosage [43]. It is designed to address common barriers to reducing polypharmacy identified in the literature, such as lack of knowledge regarding deprescribing, patient fears of not being able to restart medications, physician and patient unwillingness to initiate conversations about medication reduction, physician uncertainty about how to conduct goals of care conversations, lack of evidence support or a clear pathway for approaching polypharmacy, and a focus on providing care congruent with multimorbidity rather than disease-specific clinical guidelines [44-47]. TAPER conforms to standards for high quality acronyms (score 14.5) [48]. The model development and its theoretical basis will be described in a separate publication [45]; some further details are provided in the "Methods" section.

In brief, TAPER is a structured operationalized clinical pathway for comprehensive multidisciplinary collaborative medication review by the team including pharmacists and physicians in partnership with patients. The clinical pathway is structured around the core functions and principles of the primary care model and focuses on reducing medication burden, and through this, potentially the harms of unnecessary polypharmacy. TAPER is designed to explicitly overcome known barriers to polypharmacy reduction which includes patient fears of not being able to restart medications, physician/pharmacist and patient unwillingness to initiate conversation, physician/pharmacist uncertainty about how to conduct goals of care conversations, lack of evidence support or a clear pathway, lack of confidence in tapering and deprescribing, lack of teamwork, and lack of a mode for communication between pharmacist and physician [45]. The mapping of these is described explicitly and in more detail in a paper describing the theoretical model for TAPER [40]. TAPER sequentially links consultations with first a pharmacist and then a family physician and frames the medication reduction pathway as a structured "pause and monitor" drug holiday period. Evidence support tools are integrated within an underpinning secure digital platform (TaperMD) [49] to automatically flag potentially inappropriate medications and cumulative medication burdens. Patient priorities are explicitly sought across several domains. TaperMD serves to integrate the consultation elements, patient priorities and preferences, and evidence supports across providers in a shared electronic record. This platform is designed for incorporation into clinicians' existing electronic medical record systems.

\section{Methods \\ Aims}

We will use an RCT to assess the effects of implementing the TAPER model in addressing polypharmacy and on a range of health-related outcomes and health service use, in adults 70 years of age or older in a primary care setting. A secondary aim is to qualitatively explore the experience of reducing medications using this approach for patients, family physicians, and pharmacists.

\section{Research design}

We will conduct a 1:1 single blind RCT. Participants in the control group will be offered the intervention after 6-month research outcomes are assessed (wait list control).

\section{Ethics, registration, and trial guideline adherence}

At the time of submission, ethics approval was granted by the Hamilton Integrated Research Ethics Board (16November-2016; project file \#2226), the University of Alberta's Health Research Ethics Board (21-August2019; project file \# Pro00090823), and the Vancouver Island Health Authority Clinical Research Ethics Board (06-January-2020; project file \#C2019-075). The trial is registered with ClinicalTrials.gov (NCT02942927), which includes all items from the World Health Organization Trial Registration Data Set. We used the SPIRIT guidelines to inform reporting of our trial protocol (Additional File 1) [50]. We also used the TIDieR checklist for intervention description and replication (Additional File 2) [51]. Important protocol modifications will be tracked and communicated through an ethics amendment and ClinicalTrials.gov. Consent form and other related documentation given to participants and authorized surrogates available upon request.

\section{Trial setting}

The trial will take place in three Canadian primary care locations. The lead implementation site is the McMaster University Practice Based Research Network (McMaster University Sentinel and Information Collaboration) which serves a population of 50,000 patients $[1,52]$. Secondary sites will be in Alberta (Kaye Edmonton Clinic, 
over 7000 patients rostered) and British Colombia (multiple clinics in the Nanaimo Health Region).

\section{Participants}

The source population includes patients living in Canada who have a regular family physician. All patients rostered with participating family physicians are eligible for participation provided they fit inclusion and exclusion criteria.

Patients meeting the following criteria will be eligible for the trial:

- Aged 70 years of age or older

- Taking 5 or more long-term prescribed medications (excluding topical products) [7]

- Rostered to a family physician participating in the trial

- Willing to try discontinuation

Patient will be excluded based on the following exclusion criteria:

- Unable to understand English or do not have cognitive skills to understand and respond to rating scales

- Terminal illness or other circumstance precluding the 6-month study period follow-up

- Had a comprehensive medication review within the past 12 months

\section{Participant recruitment}

Patients will be identified through automated screening by participating family physicians' electronic medical record (EMR) to find patients 70 and over and taking 5 or more long-term medications. Potentially eligible patients will be sent a letter from their family physician outlining the study. If patients are interested in participating, they will be asked to either reply in a postage paid envelope to the study team or call the study phone number. They will then be contacted by a member of the study team who will go through a formal information, consent process, and eligibility screen. Patients who consent will then have the study appointments made according to their allocation to intervention or control group.

\section{Allocation and randomization}

Patients will be randomly allocated 1:1 to either the intervention or control group using an internet accessible computerized system [53]. Randomization will be blocked and stratified by site. The randomization sequence will be generated centrally using a computerized system and will be maintained external to the research team. The Biostatistics Unit at St. Joseph's Healthcare
Hamilton will be responsible for generating the sequence.

\section{Blinding}

Patients will not be blinded to the allocation as this would require preparation of identical placebos for all medications and tapering regimes and is not practically or economically feasible. This is a trial of effectiveness of reducing the number of medications, rather than a trial of the chemical effect of a drug where an identical placebo control is appropriate. The use of placebos in this planned trial would also negate the ability to look at the effects on treatment burden and medication self-efficacy that may be associated with simplification of medication regimes.

Outcome assessment will be blinded with "quarantine" methods developed in the feasibility RCT [42] to maintain blinding of the patient outcome assessor. The effectiveness of this blinding will be assessed at study end by asking the outcome assessor to guess which arm the patient is in, after outcome measures have been evaluated. Pharmacists and family physicians will be masked to allocation to some extent, in that they will not be aware of whether the appointment they are carrying out is for an intervention participant or 6-month waitlist control participant. Pharmacists and family physicians will only be able to access TaperMD and its tools for enrolled patients immediately prior to their appointments with individual patients. The data analysts will be blinded to group allocation; however, they will know the number of groups there are.

\section{Intervention}

We developed TAPER as a multifaceted intervention that integrates available evidence tools, a team approach and technology within a clinical pathway where decision making is led by consideration of patient priorities. Barriers to addressing polypharmacy identified in the literature, and in our formative work for this trial, were considered in its development [44, 46, 54-57]. Participatory-based methods were used with consumers, pharmacists and family physicians to inform the approach and development of the tool so the intervention is as "fit for purpose" and as feasible as possible. We also carried out a feasibility RCT as phase 1 of this project to inform and revise the model (reported separately) [42]. To foster fidelity, participating pharmacists and family physicians will receive an in-person training session for TaperMD and the entire intervention. A demonstration video and manual will also be provided.

TAPER will be operationalized as a structured clinical pathway in an electronic web-based platform, called TaperMD (see Additional File 3 for details), which records and integrates the following: 
1. Patient priorities for treatment: Explicit recording of patient preferences and priorities occurs first in TaperMD. Question domains cover symptom priorities, functional priorities, medication experience and preferences including ranking of the importance of symptomatic and preventive treatments, financial challenges around medications, and perceived medication burden $[40,41,56]$.

2. Patient characteristics relevant to medications including falls history, blood pressure, and renal function (may be reported by patient, pharmacist, or physician).

3. Patient medications (prescribed and over the counter), indication, including explicit questions for each medication from the pharmacist on any selfreported perceived side effects.

4. Evidence-based screen for potentially inappropriate medications: The medications recorded in \#3 (Patient medications) will be integrated into an automatic electronic screen for flagging potentially inappropriate medications or cumulative medication burdens in older adults. These flags will detail the underpinning evidence or recommendation and provide access to any available evidence tools at the "point of care." This evidence is supportive rather than prescriptive, as the model is designed to be led by the patient's priorities and preferences for treatment, as well as their experience of medication benefits and side effects. These tools and lists highlight potentially inappropriate medicines (and reasons) in older adults in a simultaneous multi-drug view consistent with a multimorbidity approach. Specific medication dimensions flagged will include standard interaction checking, potentially inappropriate medicines in older adults drawn from assessment of a wide range of jurisdiction specific lists [10], drugs contributing to anticholinergic burden score, QT prolonging drug burden, hypotensive drug burden, serontonergic drug burden, and deprescribing guidelines and algorithms where these are available. These are described in detail in Additional File 3.

5. Recommendations for drug classes around tapering approaches, potential medication withdrawal effects, and suggested monitoring parameters and time frames will be provided to support tapering and/or deprescribing.

6. One of the barriers to collaboration between clinicians is often the absence of communicating electronic medical records between providers (in particular pharmacists and prescribers). TaperMD serves as a shared record for all clinicians in the pathway to access and review. It is designed to be integrated with the electronic medical record of both pharmacists and family physicians, other relevant clinicians through an application programming interface (API) to an EMR, or printed for integration with paper records of clinicians and patients. It has been designed to also allow for future patient access.

\section{Operational steps}

\section{STEP 1: Collection of patient information}

Patient priorities and any available data on patient characteristics relevant to medications will be entered into TaperMD. For the study, this will be done by the research assistant along with the collection of baseline outcome data; however, the tool is designed for future patient/family access to complete this step (Fig. 1).

\section{STEP 2: Pharmacist consultation}

The patient will meet first with the pharmacist to review the list of medications for reconciliation with what the patient reports they are taking, including accuracy of dose and frequency and indications. Specific side effect inquiry is recorded for each medication as well as any further relevant patient characteristics. A medication review will be done, focused on medications suitable for stopping or dose reduction, supported by the TaperMD screen and evidence resources for tapering, the reported medication side effects from the patient, and review of the patient's medication experience and priorities and preferences for treatment.

The pharmacist will make initial recommendations focused on medications suitable for discontinuation or dose reduction and record these in TaperMD, which will then be visible to the family physician. The summary of this initial plan will also be integrated into the pharmacist's electronic record.

\section{STEP 3: Family physician consultation}

Within a maximum of 2 weeks later, the patient will have an appointment with their family physician to discuss the pharmacist recommendations and identify medications that may be suitable for a "pause and monitor" phase of discontinuation or dose reduction. The family physician can access and view the TaperMD record prior to the consultation (patient priorities, patient characteristics relevant to medications, evidence screen, recorded potential medication side effects, and pharmacist recommendations for each medication). The family physician will verbally review the patient's priorities and preferences, and incorporate their knowledge of the patient over time, their context, and their medical conditions in this review of the pharmacist's recommendations and discussion with the patient. Patient goals and priorities can be adjusted at this point, based on these discussions. 


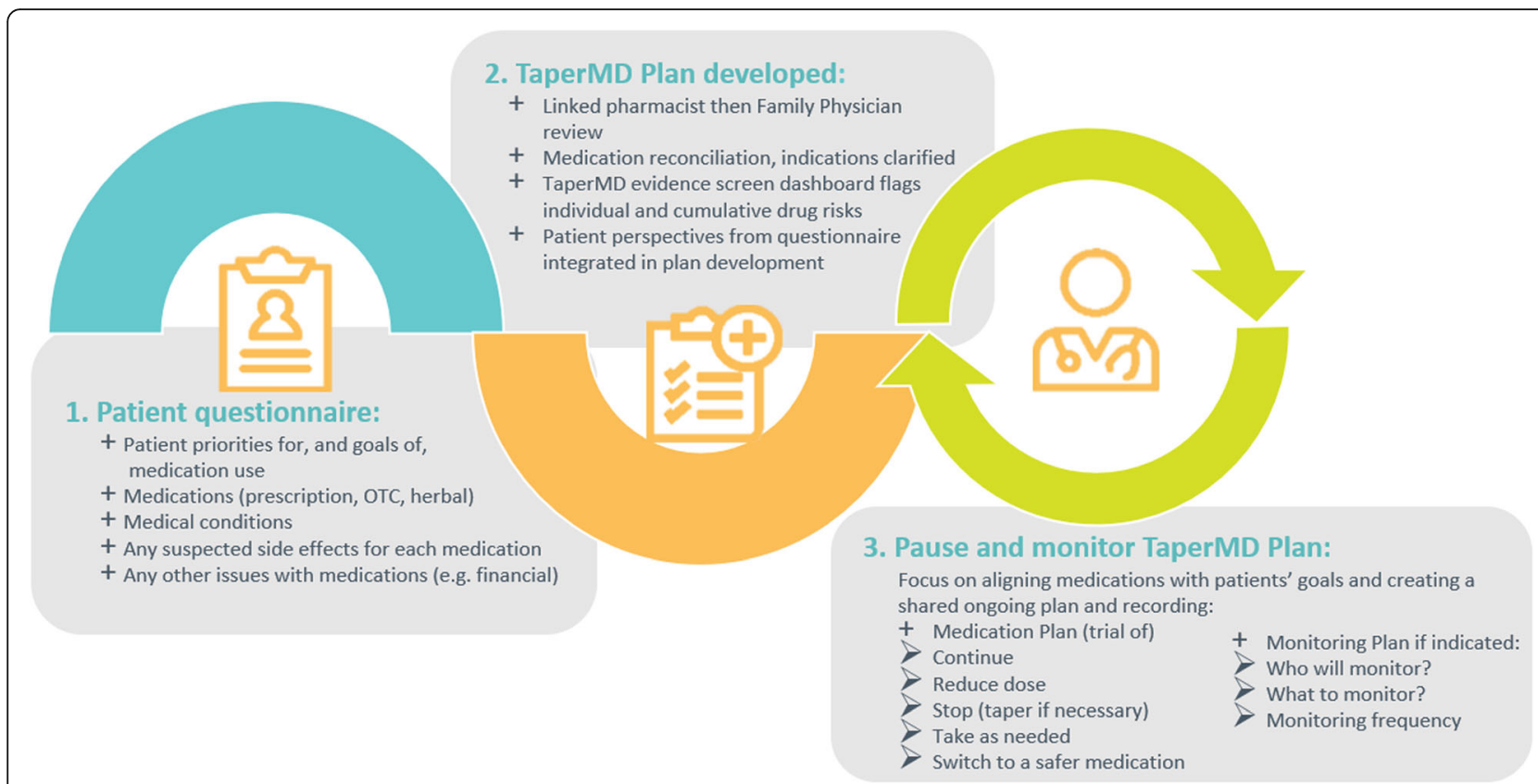

Fig. 1 Operational steps of TaperMD

Every medication is reviewed and a final recommendation is recorded to either continue, stop, reduce the dose, or switch to a safer medication. The discontinuation and follow-up monitoring plan is finalized including timing of changes (not all may occur immediately if a number are being stopped), tapering and any agreed criteria for restarting. TaperMD generates a summary of the plan (a TAPER plan), including the tapering, monitoring, and follow-up plan. This will be pulled into the usual clinical record (e.g., EMR) as the visit record and can be printed for the patient and their family.

Due to the COVID pandemic, patients were given the option to have in person or virtual/video consultations.

\section{Monitoring}

Study participants will be followed for 6 months. Participants will attend monitoring visits, as clinically indicated, with the most appropriate clinician during the "pause and monitor" phase and informed by the tools supporting tapering and monitoring. Planned monitoring visits will be recorded in TaperMD and pulled into the EMR as the visit record. Participants will have no restrictions on other clinical activities.

\section{Control group}

The control group will receive usual clinical care. The only restriction is there will be no other planned medication reviews performed in the primary care setting during this phase. Patients carry information requesting no routine medication deprescribing settings for the 6- month period, except if medically indicated. Control patients will be offered the intervention after their 6month outcome assessment. This comparator was chosen as it was felt this approach was likely to reduce contamination bias and maximize recruitment.

\section{Data collection \\ Patient characteristics}

Patient characteristics that include age, gender, household income, ethnicity, and education will be collected at baseline. Also, a measure of comorbidity [58], medication risk [59], frailty [60], and beliefs about medicines [61] will be completed. Patient preferences, priorities, and goals are recorded as part of the clinical intervention.

\section{Outcome measures}

Primary and secondary outcomes will be measured at $T_{0}$ and $T_{6}$ for effectiveness analyses (Research Questions 1 and 2, Fig. 2). See Additional File 4 for a detailed description of the outcome measures and how they relate to the data analysis plan respectively, and Additional File 5 for the detailed schedule of all data collection. Choice of measures was guided by recommendations from an international consensus that identified a set of outcomes specific for multimorbidity patients [62], evidence of their relationship to polypharmacy $[8,9,12]$, and piloting of a number of measures in our feasibility RCT (reported elsewhere) [42]. 


\section{Primary outcome measure}

The primary outcome includes the number of prescribed medications per patient at baseline and 6 months (see Additional File 4 for definition). Locally acting topical agents without substantial systemic absorption or effects are not included. Acute short-course medications such as antibiotic prescriptions are not included in the counts. This will be ascertained by a pharmacist by performing a medication reconciliation using dispensing information, the electronic medical record, and patient information. Discrepancies will be resolved by discussing with the patient and dispensing pharmacist.

\section{Secondary outcome measures}

Unless specified, all outcome measures are recorded at baseline and 6 months (see Additional File 4). Constructs included as secondary outcomes include disease burden, treatment burden, quality of life, cognition, fatigue, nutritional status, physical function capacity and ability, falls, pain, sleep, patient enablement, medication selfefficacy, and healthcare utilization.

Other medication-related information will be recorded to identify and describe the processes of medication change during the intervention. Simple descriptive data will be reported on the following process measures:

- Number of medications with persistent discontinuation at 6 months

- Number of medications re-initiated

- Number of medications switched to safer medication,

- Number of medications with dose change (decrease), and dose change (increase)

- Number of medications initiated

- Number of recommended medication changes

- Number and description of medication changes aligned with patient priorities and goals

TaperMD includes a prompt for "completed status" for every medication that includes all these options. Data will be collected through an audit of TaperMD records and progress notes, EMR, and dispensing records. We will describe any observed patterns in groupings of discontinued medications, in particular among those associated with adverse drug reactions causing hospital admissions.

\section{Adverse events, harms, and beneficial withdrawal effects} Adverse events potentially related to medication withdrawal, potential medication side effects unmasked by discontinuation (i.e., symptom relief), new or worsening side effects, and emergency department visits will be collected by solicited inquiry as well as spontaneous patient or clinician report. The definition from the Common
Terminology Criteria for Adverse Events (CTCAE) of Severe/Serious Adverse Event will be used in this study: Any event that requires in-patient hospitalization or prolongation of existing hospitalization, causes congenital malformation, results in persistent or significant disability or incapacity, is life-threatening, or results in death [63]. All adverse events and harms will be summarized and reported with the trial results.

\section{Check-in data collection}

To facilitate accurate recall of adverse events, healthcare utilization, and changes in symptoms or side effects throughout the study duration, telephone check-ins will occur at 1 week, 3 months, and 6 months post-baseline to enquire specifically about these items. Details of any adverse effects identified at these data collection points will be provided in real time to the family physician and pharmacist to address as clinically appropriate. The research ethics board will be notified of any adverse events felt to be study related.

\section{Patient and provider experience of TAPER}

Patients in the intervention arm of the study will be invited to participate in semi-structured interviews to explore the meaning of current medication practices in the context of their everyday lives and their experience of the deprescribing process. Those who respond and consent to this portion will participate in the qualitative study. Attention will be paid to the biopsychosocial experiences of patients in relation to their experiences of the intervention. We will conduct in-depth interviews at the initial stages of the intervention as well as at the end to get a full picture of their experience with the intervention and process of describing.

In addition, this subset of consenting patients will be asked to keep a diary on their chronic disease management practices, and their experience going through the intervention during the study. The aim is to understand both the experience of the intervention and any effects on the complex work of managing multiple chronic diseases. Research on patient compliance suggest that electronic diaries are effective and reliable sources of data [64]. Patients will also be asked about their satisfaction with the intervention process and with their care around medications using Likert type scales, as well as free text responses of the strengths and weaknesses of the process and whether or not they would recommend the intervention to a family member or friend.

Participating pharmacists and family physicians will be asked to record their experiences and feelings about the deprescribing process as field notes during the study. Semi-structured interviews will be conducted at the end of the study at all sites to gain a more in-depth understanding of their experience of the intervention. A 


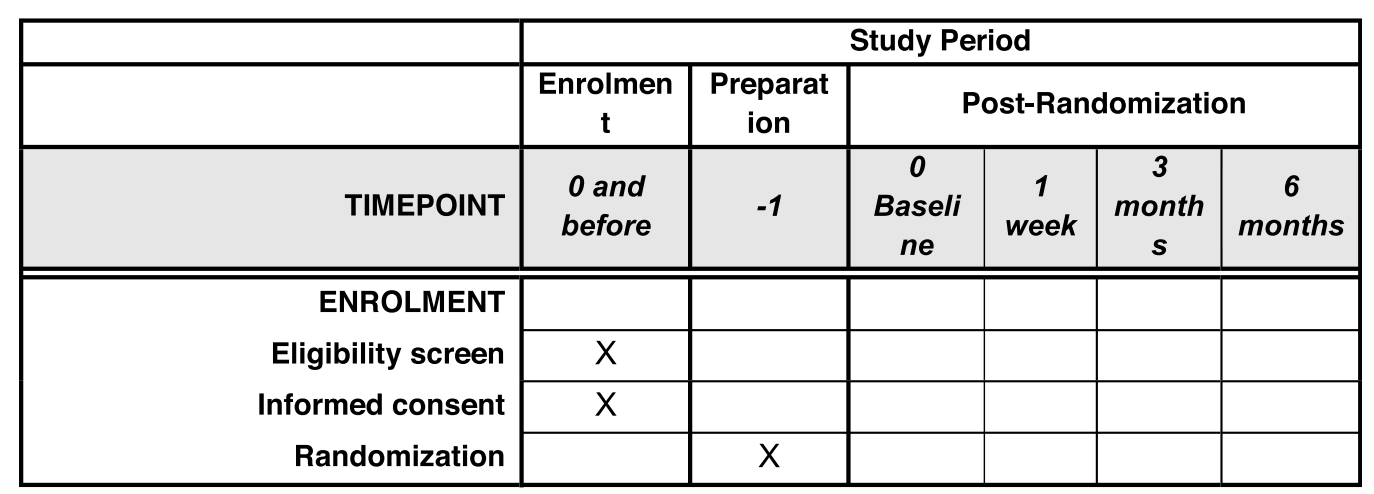

INTERVENTIONS:

\begin{tabular}{|r|l|r|l|l|l|l|}
\hline Intervention Group (TAPER) & & & & & \\
\cline { 4 - 6 } Control Group (Usual Care) & & & & & \\
\hline
\end{tabular}

ASSESSMENTS:

\begin{tabular}{|c|c|c|c|c|}
\hline Outcomes & & & & \\
\hline Number of Medications* & $\mathrm{X}$ & & & $\mathrm{X}$ \\
\hline Disease Burden** & $\mathrm{X}$ & & & $\mathrm{X}$ \\
\hline Treatment Burden** & $\mathrm{X}$ & & & $\mathrm{X}$ \\
\hline Quality of Life ${ }^{* *}$ & $\mathrm{X}$ & & & $\mathrm{X}$ \\
\hline Cognition ${ }^{* *}$ & $\mathrm{X}$ & & & $\mathrm{X}$ \\
\hline Fatigue $^{* *}$ & $\mathrm{X}$ & & & $\mathrm{X}$ \\
\hline Nutritional Status ${ }^{* *}$ & $\mathrm{X}$ & & & $\mathrm{X}$ \\
\hline $\begin{array}{r}\text { Physical Function Capacity and } \\
\text { Ability** }\end{array}$ & $\mathrm{X}$ & & & $\mathrm{X}$ \\
\hline Falls ${ }^{* *}$ & $\mathrm{X}$ & & & $\mathrm{X}$ \\
\hline Pain** & $\mathrm{X}$ & & & $\mathrm{X}$ \\
\hline Sleep** & $\mathrm{X}$ & & & $\mathrm{X}$ \\
\hline Patient enablement ${ }^{* *}$ & $\mathrm{X}$ & & & $\mathrm{X}$ \\
\hline Medication Self-Efficacy** & $\mathrm{X}$ & & & $\mathrm{X}$ \\
\hline Healthcare Utilization** & $\mathrm{X}$ & & & $\mathrm{X}$ \\
\hline Change in side effects ${ }^{+}$ & & $\mathrm{X}$ & $\mathrm{X}$ & $\mathrm{X}$ \\
\hline $\begin{array}{r}\text { Number of medications with } \\
\text { persistent discontinuation at } 6 \\
\text { months }{ }^{+}\end{array}$ & & & & $X$ \\
\hline Number of medications re-initiated ${ }^{+}$ & & & & $\mathrm{X}$ \\
\hline $\begin{array}{r}\text { Number of medications switched to } \\
\text { a safer medication }\end{array}$ & & & & $\mathrm{X}$ \\
\hline $\begin{array}{l}\text { Number of medications with dose } \\
\text { change, decrease and increase }\end{array}$ & & & & $\mathrm{X}$ \\
\hline Number of medications initiated ${ }^{+}$ & & & & $\mathrm{X}$ \\
\hline $\begin{array}{r}\text { Number of recommended } \\
\text { medication changes }\end{array}$ & & & & $\mathrm{X}$ \\
\hline $\begin{array}{l}\text { Number and description of } \\
\text { medication changes aligned with } \\
\text { patient priorities and goals }{ }^{+}\end{array}$ & & & & $X$ \\
\hline
\end{tabular}

Fig. 2 SPIRIT figure with study timeline and data collection time points 
single-item question will also be used to measure one's confidence in their own ability to undertake a process of deprescribing with their patients using a 5-point Likert scale developed for the study, as well as an open-ended question to describe the five best and five worst aspects of the intervention. A validated scale that maps to Normalization Process Theory, the NoMAD survey, is a 23-item survey that will be used to measure implementation processes from the perspectives of those directly involved in implementation of the intervention as the study progresses $[65,66]$.

\section{Economic analysis}

A 6-month trial-based economic evaluation will be conducted to compare the costs and quality-adjusted life years (QALYs) associated with the intervention and control groups (Research Question 3). Costs will be derived from health care resource utilization data collected in the case report forms and the medical records (e.g., hospitalization, emergency room visits, medication use, home care services, physiotherapy, home care visits). Fixed and variable costs associated with developing and implementing the intervention will be based on trial data. In the base case analyses, Ontario unit costs will be used to cost the healthcare resource utilization and the program costs. To calculate the QALYs, the health utility scores derived from the EQ-5D-5L questionnaire using the Canadian algorithm [67] will be weighted by time spent in health states using an area-under-thecurve approach. Healthcare resource utilization and EQ5D-5L data will be collected at baseline and at 6 months. Techniques to deal with missing data and censored data will be considered as appropriate [68]. Bootstrap techniques will be used to deal with sampling uncertainty and cost-effectiveness acceptability curves will be used to determine the probability of the intervention to be cost-effective at different willingness-to-pay thresholds (\$50,000 or $\$ 100,000 / \mathrm{QALY}$ gained). The economic evaluation will be conducted according to Canadian [69] and international [68] guidelines and from a Ministry of Health perspective. Because the duration of the study is 6 months, there is no discounting of costs or effects (QALY's).

\section{Data management}

Table 1 outlines the proposed data analysis methods as they correspond to research questions, hypotheses, and outcome measures. Where appropriate, large font showcards will be used by the researcher to visually present answer options for surveys. Most survey responses will be recorded by the researcher in Research Electronic Data Capture (REDCap) [53, 70], an electronic data capture tool hosted by The Research Institute of St. Joe's Hamilton, which allows for secure online data entry from multiple sites into a central data repository. Some data that is also relevant to the clinical assessments is recorded in TaperMD and later imported into REDCap. These processes were trialed in our feasibility study [42]. The database has automatic checks and warnings for missing data and will have regular checks for missing data and outliers among data for all sites. Clinical and patient characteristic data from TaperMD will also be imported into REDCap. These processes were tested in our feasibility study [42]. No patient identifiers will be entered into either TaperMD or REDCap, both of which are compliant with both federal and provincial Canadian privacy laws. Patient identifiers will be kept strictly confidential; electronic files containing identifiers will be encrypted and password protected and held in a secure network. Paper documents containing patient identifiers will be held in a locked filing cabinet in a locked room accessible only to members of the study team. Electronic and paper documents will be kept for 10 years.

\section{Sample size}

Sample size calculations for the RCT were performed by the team biostatistician (LT). We used data from our feasibility study to estimate sample size: mean number of medications of 7.7, a post-intervention reduction in mean (standard deviation) of $4.2( \pm 2.5)$. There was consensus from the expert international polypharmacy advisory group that a mean reduction of 2 medications is clinically important [42].

A sample size of at least 160 per group is sufficient to detect a difference in mean number of medications of 2 , assuming a starting mean of 7 and a static control group (power set at $80 \%$, significance level $5 \%$ ). It will also allow detection of an absolute difference in the proportion of patients with drug-related side effects of $15 \%$ (10\% versus $25 \%$ ) and provides adequate power for subgroup analysis for the primary outcome if proportions are $40 \%$ or more. Allowance has been made for $10 \%$ attrition; therefore, the final minimum recruitment target is 180 per group (total $N=360$ ).

\section{Quantitative data analysis}

The analysis and reporting of the results will follow the CONSORT guideline [71]. The process of patient selection and flow throughout the study will be summarized using a flow diagram. The analysis results of patient characteristics and baseline outcome variables (both primary and secondary) will be summarized using descriptive summary measures: expressed as mean (standard deviation) or median (minimum, maximum) for continuous variables depending on the distribution, and the number (percent) for categorical variables [72]. We will adopt an intention-to-treat (ITT) principle as our primary approach to analyze all outcomes and the people 
Table 1 Data analysis plan

Research question
What is the effect of a
structured medication
discontinuation clinical
pathway designed to reduce
polypharmacy on mean
number of medications and
patterns of discontinuation
compared to usual practice?

What is the effect of a structured medication discontinuation clinical pathway designed to reduce polypharmacy on patient quality of life, cognition, mobility-related fatigue, nutritional status, physical function capacity, pain, sleep, patient enablement, medication selfefficacy, medication confusion, grip strength, falls and adverse events, and hospital admissions compared to usual practice?
What is the cost-effectiveness of the structured medication discontinuation clinical pathway designed to reduce polypharmacy?

What is the experience of patients as they go through a structured medication discontinuation clinical pathway designed to reduce polypharmacy?
Not applicable
Improved disease and treatment burden, quality of life, cognition, fatigue, nutritional status, physical function capacity and ability, pain, sleep, patient enablement, medication selfefficacy and lower/fewer falls, healthcare utilization, and adverse events will be reported in the intervention arm compared to the control arm at 6-months

Hypothesis
Reduction in mean number of
medications
Improved disease and
treatment burden, quality of
life, cognition, fatigue,
nutritional status, physical
function capacity and ability,
pain, sleep, patient
enablement, medication self-
efficacy and lower/fewer falls,
healthcare utilization, and ad-
verse events will be reported in
the intervention arm compared
to the control arm at 6-months

Outcome measure

Mean number of medications (primary outcome)

Proportion of participants with $T_{6}$ successful reduction in medication number or dose

Composite variable of mean number of medication discontinuations and/or dose reductions

Disease burden
Treatment burden
Quality of life
Cognition
Fatigue
Nutritional status
Physical function capacity and
ability

To

Number of Falls

Pain

Sleep

Patient enablement

Medication self-efficacy

Healthcare resource utilization (number of hospitalizations, emergency department visits, primary care visits, proportion of patients with at least one hospitalizations)

Number of Serious adverse events and beneficial withdrawal

Cost per QALY

Lived experience with deprescribing process

Satisfaction with the intervention (5-point Likert scale)

Satisfaction with care around medications (5-point Likert scale)

Strengths and weaknesses of intervention

Lived experience with deprescribing process

Timin
$T_{0}$,
$T_{6}$

$T_{6}$

$T_{0}, T_{6}$

$T_{0}, T_{6}$

$T_{0}, T_{6}$

$T_{0}, T_{6}$

$T_{0}, T_{6}$

$T_{0}, T_{6}$

$T_{0}, T_{6}$

$T_{0}, T_{6}$

$T_{0}, T_{6}$

$T_{0}, T_{6}$

$T_{0}, T_{6}$

$T_{0}, T_{6}$

$T_{0}, T_{6}$

$T_{0}, T_{6}$

$T_{0}, T_{6}$

$T_{0}, T_{6}$

$T_{0}, T_{6}$

$T_{0}, T_{6}$

$T_{0}, T_{6}$

$T_{0}, T_{6}$

$T_{1}, T_{3}, T_{6}$

$T_{6}$

Cost utility analysis

Linear regression

Logistic regression
Method of analysis

Linear regression

Linear regression

Linear regression

Linear regression for all continuous outcomes, Logistic regression for Categorical outcomes and Count data models (e.g., Poisson/negative binomial regression, zeroinflated models) for count outcomes
What are the experiences of the pharmacist and family physician of managing patients through the deprescribing
Not applicable
$T_{6}$

$T_{6}$

$T_{0}, T_{6}$

$T_{6}$

$T_{6}$
Descriptive analysis of Semistructured interview/patient diaries

Descriptive statistics (M, SD)

Linear regression

Descriptive analysis

Thematic analysis of semistructured interviews/field notes 
Table 1 Data analysis plan (Continued)

\begin{tabular}{|c|c|c|c|c|}
\hline Research question & Hypothesis & Outcome measure & Timing & Method of analysis \\
\hline \multirow[t]{3}{*}{ process? } & & $\begin{array}{l}\text { Confidence in medication } \\
\text { discontinuation (5-point Likert } \\
\text { scale) }\end{array}$ & $T_{0}, T_{6}$ & Linear regression \\
\hline & & $\begin{array}{l}\text { Five best/worst aspects of } \\
\text { intervention }\end{array}$ & $T_{6}$ & Descriptive analysis \\
\hline & & Implementation processes & $\begin{array}{l}\text { Early/late } \\
\text { implementation }\end{array}$ & Descriptive analysis \\
\hline
\end{tabular}

$T_{0}$ baseline, $T_{3}$ month $3, T_{6}$ month 6

doing the analysis will be blind to study group allocation. Assuming data will be missing at random (MAR), we will also use multiple imputation [MI] through chained equations to impute missing data [73]. In total, five datasets will be imputed and pooled effect estimates (odds ratios for categorical outcomes, mean difference for continuous outcomes, and rate ratio for count outcomes) along with 95\% confidence intervals and the associated $p$ values will be reported. All statistical tests will be twosided at the 0.05 level of significance. $P$ values will be reported to three decimal places with values less than 0.001 , reported as $<0.001$. We will not adjust this for multiple analyses for secondary outcomes as we are considering them simply as exploratory. The patient will be the unit of analysis. Adopting an ITT principle, we will use linear regression to analyze both primary and secondary continuous outcomes. Analysis will be blinded. For categorical outcomes, we will use logistic regression. Count data models (e.g., Poisson/negative binomial regression, zero-inflated models) will be used to analyze the count outcomes. We will perform pre-specified subgroup analyses by adding an interaction term between intervention groups and the following subgroups: (1) age ( $\geq 85$ versus $<85$ years); and (2) frailty (frail versus severely frail, based on Rolfson et al., 2006 [60]). The interaction tests will be completely exploratory in nature and not adjusted for multiple testing. We examined articles in our review of reviews [74] of interventions to address polypharmacy in older adults living with multimorbidity that may have contained sub-analyses for age and frailty. We found one systematic review that contained a sub-analysis for mortality associated with deprescribing intervention subgroup analyses based on age [75]; and concluded there was no effect. Thus, we hypothesize that there will be no significant interaction effects for either sub-analysis. The interaction tests will be exploratory in nature and not adjusted for multiple testing. We will run sensitivity analyses to assess the robustness of the primary results, adopting a per-protocol approach. All analyses will be performed using SAS software 9.4 (Cary, NC).

We aim to book the family physician's appointment within 1 week with a maximum of 2 weeks from the pharmacist appointment but acknowledge this may be difficult due to busy physician schedules or patient commitment changes. We aim to perform another sensitivity analysis to account for these differences in the timeline that may occur through linear regression.

\section{Qualitative data analysis}

As some decisions are often emergent in qualitative research, we provide only brief details of our analytic approach to the qualitative piece to the study. Pseudonyms will be assigned to participants. Audiotapes for interviews will be transcribed verbatim. The transcripts will be coded inductively and independently by two research team members in three stages: open, axial, and selective [76]. The interview data generated (with patients, pharmacists, and family physicians), family physician and pharmacist field notes, and patient diaries will be analyzed using thematic analysis to identify, analyze, and report patterns in the data. Dedoose, an online data analysis application for qualitative and mixed methods research [77], will be used to support analysis. Consistent with recommendations, two strategies will be used to foster trustworthiness [78, 79]. First, two coders will independently code $100 \%$ of the data and meet to discuss initial patterns and themes and explore disagreement. Second, authenticity will be maintained by displaying direct quotations within the results.

\section{Adherence and fidelity issues}

Adherence will not affect the ability to measure the main study outcomes in the way it does in other trials as it is part of the outcome measure itself. The need to restart medications is not seen as a protocol violation or bias, nor is it seen as a failure of treatment, rather it is seen as effective monitoring during the "pause and monitor" phase. Fidelity to the intervention will however be assessed using a checklist of critical elements (see Additional File 6).

\section{Contamination}

Access to TaperMD is by individual record unique ID. Pharmacists and physicians will only be able to access TaperMD intervention patients. Access will only be 
available for control patients after they have passed through the 6-month data collection point.

\section{Loss to follow-up}

We conservatively anticipate the loss to follow-up rate to be a maximum of $10 \%$. Our experience in a discontinuation RCT of antidepressants in primary care showed only a $4 \%$ loss to follow-up over 18 months [80], and our feasibility studies in the same age group showed a loss to follow-up of less than $8 \%$. Our TAPER feasibility RCT showed loss to follow-up of only 3 patients. We found regular contact, multiple alternate contact details, and a measured informed consent process, particularly ensuring participants are clearly able to be available for follow-up, are helpful in minimizing such losses. In our feasibility study, we found it unhelpful to recruit participants during the "snowbird" period in winter who were traveling outside of Canada for an extended time as many older Canadians do. Perceived insurance restrictions mean they are not willing to change their medication in the period prior to leaving. The participants lost to follow-up will be classified as lost to follow-up due to an inability to contact, elected to no longer be part of any further follow-up (withdrawal of consent), or unable to participate because of ill health. In cases where participants who are lost to follow-up do not explicitly withdraw consent, medication and health care utilization data will still be collected from patient charts via chart audit where possible.

\section{Safety and monitoring}

Details of any clinically adverse symptoms or effects reported to researchers at research data collection points will be securely provided to their family physician and pharmacist at that time, either by personal communication from the research team or electronically to a secure clinic fax number as appropriate. These processes did not compromise the blinding in our feasibility study [42]. Any such adverse effects will be addressed within the usual primary care clinical setting by their family physician who will be responsible for the clinical followup medication pause or dose reduction. Clinical followup will occur as determined by the monitoring requirements for the particular drugs discontinued.

If the patient experiences a serious adverse event (SAE), defined in a previous section on adverse events, a local SAE record will be completed and reported to the family physician within $24 \mathrm{~h}$ if they are unaware. The study team will notify the research ethics board of any SAE thought to be related to the study, using the standard SAE form. The form will be signed off by the principal investigator within $48 \mathrm{~h}$. No formal auditing of trial conduct will take place. There will not be a data monitoring committee involved with the trial as there are no stopping rules for the trial and no planned interim analyses. Research staff will be responsible for overseeing study progress. Regular contact among study team members and clinic staff will be made to discuss and resolve any issues thought to be related to study conduct, and if any protocol or process changes are required these will be recorded as previously stated. There are no provisions for post-trial or ancillary care, as patients will continue to receive care as necessary from their physician throughout and following participation the trial. Furthermore, the study is low-risk and no harms from trial participation are anticipated.

\section{Dissemination policy}

All results of the trial will be published, and there are no publication restrictions.

\section{Discussion}

This study protocol describes an RCT designed to assess the effect of TAPER in reducing polypharmacy. Polypharmacy is associated with negative health outcomes. It is not known whether these effects are reversible if polypharmacy is reduced. The study is designed to assess the effect of TAPER on reducing polypharmacy and the extent to which this affects selected health outcomes, based on the negative associations with polypharmacy found in non-randomized studies. This study will be a large multi-centered single-blinded RCT.

Our study so far is unique in its design in aiming to introduce an operationalized structured clinical pathway aimed to tackle polypharmacy in a primary care setting while at the same time recording patient's goals and priorities for treatment. A variety of explicit tools and deprescribing guidelines are available to flag medications that may be inappropriate or suitable for discontinuation in older adults. In addition, there are several studies evaluating pharmacist and physician collaboration to improve medication management in primary care [18]. However, very few pathways integrate all of these in one place and across all medications simultaneously, and fewer operationalize patients' views and consideration of patients' actual goals and priorities for treatment.

A strength of this trial design is its pragmatic nature and the setting where the evidence will be applied. The pathway and tool are immediately scalable and the results of the trial, if successful, will be directly translatable into clinical practice. Evidence from this study will help understand the provision of a systematic approach to considering reduction of the burden of polypharmacy, as well as providing a systematic clinical pathway for implementation including integration of available evidence tools, active monitoring, and reinstatement criteria.

While there are many studies associating negative health outcomes with polypharmacy, these result in 
observational data only and do not prove that outcomes improve when polypharmacy is reduced. If successful, our aim in testing this intervention is to lay the foundation for this model to be incorporated and become a part of routine preventive care in older adults.

\section{Trial status}

Recruitment for the trial started in June, 2018, and is expected to continue recruitment until late 2021. This is protocol version 1 , July 31, 2020.

\begin{abstract}
Abbreviations
API: Application programming interface; CTCAE: Common Terminology Criteria for Adverse Events; EMR: Electronic medical records; ITT: Intention-totreat; LOCF: Last-Observation-Carried-Forward; MAR: Missing at random; MI: Multiple imputation; MMSE: Mini-Mental Status Examination; MNAS F: Mini-Nutritional Assessment Short-Form; PEl: Patient Enablement Instrument; PIM: Potentially inappropriate medication; RCT: Randomized controlled trial; REDCap: Research Electronic Data Capture; SAE: Serious adverse event; SEAMS: Self-Efficacy for Appropriate Medication Use scale; STOPP: Screening Tool of Older Person's Prescriptions; TAPER: Team Approach to Polypharmacy Evaluation and Reduction; QALY: Qualityadjusted life years
\end{abstract}

\section{Supplementary Information}

The online version contains supplementary material available at https://doi. org/10.1186/s13063-021-05685-9.

Additional file 1. PDF. Spirit 2013 Checklist.

Additional file 2. PDF. TIDier Checklist.

Additional file 3. PDF. TaperMD. A list of the questions on the TaperMD web-based platform.

Additional file 4. PDF. Quantitative patient outcomes measure descriptions. A list of the primary and secondary outcomes, descriptions of the measures and the psychometric properties of the measures.

Additional file 5. PDF. Data collection schedule. A diagram outlining the proposed timeline of the study.

Additional file 6. PDF. Site fidelity to TAPER-RCT model. The checklist that will be used to measure a site's adherence to the TAPER-RCT protocol.

\section{Acknowledgements}

We would like to acknowledge the contributions of the family physicians, nurse practitioners, and patients of the McMaster Family Health Team, in carrying out the feasibility study and providing feedback on tool and pathway development. Thanks to the management and administration teams of the McMaster Family Health Team clinics for facilitating and supporting the feasibility study that informed the development of this protocol. Special thanks to the pharmacists Kristina Frizzle, Jane JurcicVrataric, and Kiska Colwill for testing the operationalization of the pathway and tools. Thanks to Jennifer Lawson for assistance in automatic approaches to identifying potentially eligible participants in electronic medical records.

\section{Authors' contributions}

All authors contributed to the design and drafting of the protocol. DM conceptualized the study, pathway model design, and underpinning tool. DM and LL drafted the protocol and led the feasibility and other studies on which it is based. DS led the systematic review of evidence for effectiveness of different approaches to polypharmacy. JET and DO will lead the economic evaluation and JG will lead the qualitative analyses, with support from MS. LT is the lead consultant on biostatistical and RCT design aspects, along with SB who will perform the analyses. JT is the patient coinvestigator and leads consumer advice on the pathway, tools, and intervention conduct. JJ, KC, LD, $\mathrm{BF}$, and ND led the conceptualization of the pharmacist role. SG is the lead investigator in Alberta and JM and AC in British Columbia. AC will lead the study knowledge translation. JR is the lead advisor on functional assessments and $\mathrm{DM}, \mathrm{CR}$, and JT co-lead the development of goals and priorities aspects of intervention and trial design. JL, JP, KF, AA, and JT led the larger operational protocol development, assisted with data collection in the feasibility and other underpinning studies, and contributed substantially to the drafting of this protocol. SD developed study database, completed data quality checks, and provided overall oversight of database integrity. There are no plans to use professional writers. All author(s) read and approved the final manuscript.

\section{Funding}

Funding for this study was provided by a Project Grant from the Canadian Institutes of Health Research (CIHR) grant (\#PJT 148971); http://www.cihr-irsc. gc.ca/e/193.html). Study funders were not involved in decisions on study design, or collection, management, analysis, and interpretation of data, writing of the report, and the decision to submit the report for publication. The funding agency has no authority over these decisions. The Labarge Optimal Aging fund provided the funding for the initial feasibility RCT that supported this project.

\section{Availability of data and materials}

The study team will have full access to the dataset. Data will be made available once planned analyses and publications by the study team are completed, as well as online training materials. Anonymized patient-level data will be made available for meta-analyses on request. There are no plans to grant access to statistical code.

\section{Declarations}

Ethics approval and consent to participate

At the time of submission, ethics approval for the study was granted by the Hamilton Integrated Research Ethic Board on the 16-November-2016. The project file number is 2226. Ethics approval was also granted by the University of Alberta's Health Research Ethics Board on 21 Auqust 2019, file number Pro00090823; and by the Vancouver Island Health Authority Clinical Research Ethics Board on 06 January 2020, file number C2019-075. Important modifications to the protocol will be tracked and communicated through an ethics amendment and on clinicaltrial.gov. The trial was registered on clinicaltrials. gov on October 24, 2016 (NCT02942927). Written informed consent will be obtained from all participants in the study. A copy of the consent form is available upon reasonable request.

\section{Consent for publication}

Not applicable

\section{Competing interests}

The authors declare that they have no competing interests.

\section{Author details}

'Department of Family Medicine, McMaster University, 100 Main Street West., 5th floor, Hamilton, Ontario L8P 1H6, Canada. ${ }^{2}$ University of Alberta, 6-60 University Terrace, Edmonton, Alberta, Canada. ${ }^{3}$ University of Victoria, 3800 Finnerty Road, Victoria, BC, Canada. ${ }^{4}$ University of Toronto, 144 College Street, Toronto, Ontario, Canada. ${ }^{5}$ Bruyère Research Institute, 43 Bruyère Street, Ottawa, Ontario, Canada. 'University of British Columbia, 2405 Wesbrook Mall, Vancouver, BC, Canada.

Received: 25 March 2021 Accepted: 5 October 2021

Published online: 26 October 2021

References

1. Farrell B, Mangin D. Deprescribing is an essential part of good prescribing. Am Fam Physician. 2019;99(1):7-9.

2. Lazarou J, Pomeranz BH, Corey PN. Incidence of adverse drug reactions in hospitalized patients: a meta-analysis of prospective studies. JAMA. 1998; 279(15):1200-5.

3. Lipska KJ, Ross JS, Wang Y, Inzucchi SE, Minges K, Karter AJ, et al. National trends in US hospital admissions for hyperglycemia and hypoglycemia among Medicare beneficiaries, 1999 to 2011. JAMA Intern Med. 2014;174(7): 1116-24. https://doi.org/10.1001/jamainternmed.2014.1824. 
4. Malvezzi M, Bertuccio P, Levi F, La Vecchia C, Negri E. European cancer mortality predictions for the year 2013. Ann Oncol. 2013;24(3):792-800. https://doi.org/10.1093/annonc/mdt010.

5. Tinetti ME, Han L, Lee DS, McAvay GJ, Peduzzi P, Gross CP, et al. Antihypertensive medications and serious fall injuries in a nationally representative sample of older adults. JAMA Intern Med. 2014;174(4):588-95. https://doi.org/10.1001/jamainternmed.2013.14764.

6. Commission of the European Communities. Regulation of the European Parliament and of the council amending, as regards pharmacovigilance of medicinal products for human use. 2008. https://ec.europa.eu/health/sites/ health/files/files/pharmacos/pharmpack_12_2008/pharmacovigilance-iavol1_en.pdf. Accessed 23 March 2021

7. Masnoon N, Shakib S, Kalisch-Ellett L, Caughey GE. What is polypharmacy? A systematic review of definitions. BMC Geriatr. 2017;17(1):230. https://doi. org/10.1186/s12877-017-0621-2

8. Jyrkka J, Enlund H, Lavikainen P, Sulkava R, Hartikainen S. Association of polypharmacy with nutritional status, functional ability and cognitive capacity over a three-year period in an elderly population. Pharmacoepidemiol Drug Saf. 2011;20(5):514-22. https://doi.org/10.1002/ pds.2116.

9. Leipzig RM, Cumming RG, Tinetti ME. Drugs and falls in older people: a systematic review and meta-analysis: I. Psychotropic drugs. J Am Geriatr Soc. 1999:47(1):30-9. https://doi.org/10.1111/j.1532-5415.1999.tb01898.x.

10. Mangin D, Bahat G, Golomb BA, Mallery LH, Moorhouse P, Onder G, et al. International Group for Reducing Inappropriate Medication Use \& Polypharmacy (IGRIMUP): Position Statement and 10 Recommendations for Action. Drugs Aging. 2018;35(7):575-87. https://doi.org/10.1007/s40266-0180554-2.

11. Moore AR, O'Keeffe ST. Drug-induced cognitive impairment in the elderly. Drugs Aging. 1999;15(1):15-28. https://doi.org/10.2165/00002512-19991501 $0-00002$.

12. Hilmer SN, Gnjidic D. The effects of polypharmacy in older adults. Clin Pharmacol Ther. 2009;85(1):86-8. https://doi.org/10.1038/clpt.2008.224.

13. Maher RL, Hanlon J, Hajjar ER. Clinical consequences of polypharmacy in elderly. Expert Opin Drug Saf. 2014;13(1):57-65. https://doi.org/10.1517/1474 0338.2013 .827660

14. May C, Montori VM, Mair FS. We need minimally disruptive medicine. BMJ. 2009;339(aug11 2):b2803. https://doi.org/10.1136/bmj.b2803.

15. Cooper JA, Cadogan CA, Patterson SM, Kerse N, Bradley MC, Ryan C, et al. Interventions to improve the appropriate use of polypharmacy in older people: a Cochrane systematic review. BMJ Open. 2015;5(12):e009235. https://doi.org/10.1136/bmjopen-2015-009235.

16. Dills H, Shah K, Messinger-Rapport B, Bradford K, Syed Q. Deprescribing medications for chronic diseases management in primary care settings: a systematic review of randomized controlled trials. J Am Med Dir Assoc. 2018;19(11):923-35 e2. https://doi.org/10.1 016/j.jamda.2018.06.021.

17. Gnjidic D, Le Couteur DG, Kouladjian L, Hilmer SN. Deprescribing trials: methods to reduce polypharmacy and the impact on prescribing and clinical outcomes. Clin Geriatr Med. 2012;28(2):237-53. https://doi.org/10.101 6/j.cger.2012.01.006.

18. Johansson T, Abuzahra ME, Keller S, Mann E, Faller B, Sommerauer C, et al. Impact of strategies to reduce polypharmacy on clinically relevant endpoints: a systematic review and meta-analysis. Br J Clin Pharmacol. 2016; 82(2):532-48. https://doi.org/10.1111/bcp.12959.

19. Lee JQ, Ying K, Lun P, Tan KT, Ang W, Munro Y, et al. Intervention elements to reduce inappropriate prescribing for older adults with multimorbidity receiving outpatient care: a scoping review. BMJ Open. 2020;10(8):e039543. https://doi.org/10.1136/bmjopen-2020-039543.

20. Rankin A, Cadogan CA, Patterson SM, Kerse N, Cardwell CR, Bradley MC, et al. Interventions to improve the appropriate use of polypharmacy for older people. Cochrane Database Syst Rev. 2018;9(9):CD008165. https://doi. org/10.1002/14651858.CD008165.pub4.

21. Garfinkel D, Mangin D. Feasibility study of a systematic approach for discontinuation of multiple medications in older adults: addressing polypharmacy. Arch Intern Med. 2010;170(18):1648-54. https://doi.org/10.1 001/archinternmed.2010.355.

22. Ailabouni N, Mangin D, Nishtala PS. DEFEAT-polypharmacy: deprescribing anticholinergic and sedative medicines feasibility trial in residential aged care facilities. Int J Clin Pharm. 2019;41(1):167-78. https://doi.org/10.1007/s11 096-019-00784-9.
23. Curtin D, Gallagher PF, O'Mahony D. Explicit criteria as clinical tools to minimize inappropriate medication use and its consequences. Ther Adv Drug Saf. 2019;10:2042098619829431. https://doi.org/10.1177/204209861982 9431.

24. Dunn RL, Harrison D, Ripley TL. The beers criteria as an outpatient screening tool for potentially inappropriate medications. Consult Pharm. 2011;26(10): 754-63. https://doi.org/10.4140/TCP.n.2011.754.

25. Gallagher PF, O'Connor MN, O'Mahony D. Prevention of potentially inappropriate prescribing for elderly patients: a randomized controlled trial using STOPP/START criteria. Clin Pharmacol Ther. 2011;89(6):845-54. https:// doi.org/10.1038/clpt.2011.44

26. Buck MD, Atreja A, Brunker CP, Jain A, Suh TT, Palmer RM, et al. Potentially inappropriate medication prescribing in outpatient practices: prevalence and patient characteristics based on electronic health records. Am J Geriatr Pharmacother. 2009; 7(2):84-92. https:/doi.org/10.1016/j.amjopharm.2009.03.001.

27. Hamilton H, Gallagher P, Ryan C, Byrne S, O'Mahony D. Potentially inappropriate medications defined by STOPP criteria and the risk of adverse drug events in older hospitalized patients. Arch Intern Med. 2011;171(11): 1013-9. https://doi.org/10.1001/archinternmed.2011.215.

28. Hill-Taylor B, Sketris I, Hayden J, Byrne S, O'Sullivan D, Christie R. Application of the STOPP/START criteria: a systematic review of the prevalence of potentially inappropriate prescribing in older adults, and evidence of clinical, humanistic and economic impact. J Clin Pharm Ther. 2013;38(5): 360-72. https://doi.org/10.1111/jcpt.12059.

29. Hill-Taylor B, Walsh KA, Stewart S, Hayden J, Byrne S, Sketris IS. Effectiveness of the STOPP/START (Screening Tool of Older Persons' potentially inappropriate Prescriptions/Screening Tool to Alert doctors to the Right Treatment) criteria: systematic review and meta-analysis of randomized controlled studies. J Clin Pharm Ther. 2016;41(2):158-69. https://doi.org/1 $0.1111 /$ jcpt.12372.

30. O'Connor MN, O'Sullivan D, Gallagher PF, Eustace J, Byrne S, O'Mahony D. Prevention of hospital-acquired adverse drug reactions in older people using screening tool of older persons' prescriptions and screening tool to alert to right treatment criteria: a cluster randomized controlled trial. J Am Geriatr Soc. 2016;64(8):1558-66. https://doi.org/10.1111/jgs.14312.

31. Gokula M, Holmes HM. Tools to reduce polypharmacy. Clin Geriatr Med. 2012;28(2):323-41. https://doi.org/10.1016/j.cger.2012.01.011.

32. Hilmer SN, Mager DE, Simonsick EM, Cao Y, Ling SM, Windham BG, et al. A drug burden index to define the functional burden of medications in older people. Arch Intern Med. 2007;167(8):781-7. https://doi.org/10.1001/a rchinte.167.8.781.

33. Lu WH, Wen YW, Chen LK, Hsiao FY. Effect of polypharmacy, potentially inappropriate medications and anticholinergic burden on clinical outcomes: a retrospective cohort study. CMAJ. 2015;187(4):E130-E7. https://doi.org/10.1 503/cmaj.141219.

34. Bjerre LM, Farrell B, Hogel M, Graham L, Lemay G, McCarthy L, et al. Deprescribing antipsychotics for behavioural and psychological symptoms of dementia and insomnia: Evidence-based clinical practice guideline. Can Fam Physician. 2018;64(1):17-27.

35. Farrell B, Black C, Thompson W, McCarthy L, Rojas-Fernandez C, Lochnan H, et al. Deprescribing antihyperglycemic agents in older persons: evidencebased clinical practice guideline. Can Fam Physician. 2017;63(11):832-43.

36. Farrell B, Pottie K, Thompson W, Boghossian T, Pizzola L, Rashid FJ, et al. Deprescribing proton pump inhibitors: evidence-based clinical practice guideline. Can Fam Physician. 2017;63(5):354-64.

37. Pottie K, Thompson W, Davies S, Grenier J, Sadowski CA, Welch V, et al. Deprescribing benzodiazepine receptor agonists: evidence-based clinical practice guideline. Can Fam Physician. 2018;64(5):339-51.

38. Reeve E, Farrell B, Thompson W, Herrmann N, Sketris I, Magin PJ, et al. Deprescribing cholinesterase inhibitors and memantine in dementia: guideline summary. Med J Aust. 2019;210(4):174-9. https://doi.org/10.5694/ mja2.50015.

39. Steinman MA, Fick DM. Using Wisely: a reminder on the proper use of the American Geriatrics Society Beers Criteria(R). J Am Geriatr Soc. 2019;67(4): 644-6. https://doi.org/10.1111/jgs.15766.

40. Mangin D, Stephen G, Bismah V, Risdon C. Making patient values visible in healthcare: a systematic review of tools to assess patient treatment priorities and preferences in the context of multimorbidity. BMJ Open. 2016;6(6): e010903. https://doi.org/10.1136/bmjopen-2015-010903.

41. Fried TR, Tinetti M, Agostini J, lannone L, Towle V. Health outcome prioritization to elicit preferences of older persons with multiple health 
conditions. Patient Educ Couns. 2011;83(2):278-82. https://doi.org/10.1016/j. pec.2010.04.032

42. Mangin D. Team approach to polypharmacy evaluation and reduction: feasibility study of usual care versus a structured clinical pathway to reduce polypharmacy. In Preparation. 2021.

43. Mangin D, Heath I, Jamoulle M. Beyond diagnosis: rising to the multimorbidity challenge. BMJ. 2012;344(jun13 2):e3526. https://doi.org/1 $0.1136 / \mathrm{bmj} . \mathrm{e} 3526$.

44. Anderson K, Stowasser D, Freeman C, Scott I. Prescriber barriers and enablers to minimising potentially inappropriate medications in adults: a systematic review and thematic synthesis. BMJ Open. 2014;4(12):e006544. https://doi.org/10.1136/bmjopen-2014-006544.

45. Mangin D. Theoretical underpinnings of a model to reduce polypharmacy and its negative health effects: Introducing the Team Approach to Polypharmacy Evaluation and Reduction (TAPER). In Preparation. 2021.

46. Reeve E, To J, Hendrix I, Shakib S, Roberts MS, Wiese MD. Patient barriers to and enablers of deprescribing: a systematic review. Drugs Aging. 2013; 30(10):793-807. https://doi.org/10.1007/s40266-013-0106-8.

47. Wallis KA, Andrews A, Henderson M. Swimming against the tide: primary care physicians' views on deprescribing in everyday practice. Ann Fam Med. 2017;15(4):341-6. https://doi.org/10.1370/afm.2094.

48. Pottegard A, Haastrup MB, Stage TB, Hansen MR, Larsen KS, Meegaard PM, et al. SearCh for humourlstic and Extravagant acroNyms and Thoroughly Inappropriate names For Important Clinical trials (SCIENTIFIC): qualitative and quantitative systematic study. BMJ. 2014;349(dec16 13):g7092. https:// doi.org/10.1136/bmj.g7092.

49. Mangin D. TaperMD: About. 2021. https:/tapermd.com/tapering-resources/about/. .

50. Chan AW, Tetzlaff JM, Altman DG, Laupacis A, Gotzsche PC, Krleza-Jeric K, et al. SPIRIT 2013 statement: defining standard protocol items for clinical trials. Ann Intern Med. 2013;158(3):200-7. https://doi.org/10.7326/0003-481 9-158-3-201302050-00583.

51. Hoffmann TC, Glasziou PP, Boutron I, Milne R, Perera R, Moher D, et al. Better reporting of interventions: template for intervention description and replication (TIDieR) checklist and guide. BMJ. 2014;348(mar07 3):g1687. https://doi.org/10.1136/bmj.g1687.

52. Mangin D, Lawson J, Cuppage J, Shaw E, Ivanyi K, Davis A, et al. Legacy drug-prescribing patterns in primary care. Ann Fam Med. 2018;16(6):515-20. https://doi.org/10.1370/afm.2315.

53. Harris PA, Taylor R, Thielke R, Payne J, Gonzalez N, Conde JG. Research electronic data capture (REDCap)--a metadata-driven methodology and workflow process for providing translational research informatics support. J Biomed Inform. 2009;42(2):377-81. https://doi.org/10.1016/j. jbi.2008.08.010.

54. Dowden A. Deprescribing: reducing inappropriate polypharmacy. Prescriber. 2017;28(2):45-9. https://doi.org/10.1002/psb.1541.

55. Kesselheim AS, Avorn J, Sarpatwari A. The high cost of prescription drugs in the United States: origins and prospects for reform. JAMA. 2016;316(8):85871. https://doi.org/10.1001/jama.2016.11237.

56. Mangin D, Risdon C, Lamarche L, Langevin J, Ali A, Parascandalo J, et al. "I think this medicine actually killed my wife: patient and family perspectives on shared decision-making to optimize medications and safety. Ther Adv Drug Saf. 2019;10: 2042098619838796. https://doi.org/10.1177/2042098619838796.

57. Reeve $\mathrm{E}$, Wiese MD. Benefits of deprescribing on patients' adherence to medications. Int J Clin Pharm. 2014;36(1):26-9. https://doi.org/10.1007/s11 096-013-9871-z.

58. Charlson ME, Pompei P, Ales KL, MacKenzie CR. A new method of classifying prognostic comorbidity in longitudinal studies: development and validation. I Chronic Dis. 1987;40(5):373-83. https//doi.org/10.1016/0021-9681(87)90171-8.

59. Barenholtz LH. Self-administered medication-risk questionnaire in an elderly population. Ann Pharmacother. 2003;37(7-8):982-7. https://doi.org/10.1345/a ph.1C305.

60. Rolfson DB, Majumdar SR, Tsuyuki RT, Tahir A, Rockwood K. Validity and reliability of the Edmonton Frail Scale. Age Ageing. 2006;35(5):526-9. https://doi.org/10.1093/ageing/afl041.

61. Horne R, Weinman J, Hankins M. The beliefs about medicines questionnaire: the development and evaluation of a new method for assessing the cognitive representation of medication. Psychology \& Health. 1999;14(1):124. https://doi.org/10.1080/08870449908407311.

62. Smith SM, Wallace E, Salisbury C, Sasseville M, Bayliss E, Fortin M. A Core Outcome Set for Multimorbidity Research (COSmm). Ann Fam Med. 2018; 16(2):132-8. https://doi.org/10.1370/afm.2178.
63. Health Canada. Reporting adverse reactions to marketed health products guidance document for industry. 2018. https://www.canada.ca/content/da $\mathrm{m} / \mathrm{hc}$-sc/documents/services/drugs-health-products/reports-publications/ medeffect-canada/reporting-adverse-reactions-marketed-health-productsguidance-industry/reporting-adverse-reactions-marketed-health-productsguidance-industry.pdf.

64. Stone AA, Shiffman S, Schwartz JE, Broderick JE, Hufford MR. Patient compliance with paper and electronic diaries. Control Clin Trials. 2003;24(2): 182-99. https://doi.org/10.1016/S0197-2456(02)00320-3.

65. May C, Rapley T, Mair FS, Treweek S, Murray E, Ballini L, et al. Normalization Process Theory On-line Users' Manual, Toolkit and NoMAD instrument. 2015. http://www.normalizationprocess.org. .

66. May C, Finch T, Mair F, Ballini L, Dowrick C, Eccles M, et al. Understanding the implementation of complex interventions in health care: the normalization process model. BMC Health Serv Res. 2007;7(1):148. https:// doi.org/10.1186/1472-6963-7-148

67. Xie F, Pullenayegum E, Gaebel K, Bansback N, Bryan S, Ohinmaa A, et al. A Time Trade-off-derived Value Set of the EQ-5D-5L for Canada. Med Care. 2016;54(1):98-105. https://doi.org/10.1097/MLR.0000000000000447.

68. Ramsey SD, Willke RJ, Glick H, Reed SD, Augustovski F, Jonsson B, et al. Cost-effectiveness analysis alongside clinical trials II-An ISPOR Good Research Practices Task Force report. Value Health. 2015;18(2):161-72. https://doi.org/10.1016/j.jval.2015.02.001.

69. Guidelines for the economic evaluation of health technologies: Canada. 4th ed. Ottawa: CADTH; 2017 Mar.

70. Harris PA, Taylor R, Minor BL, Elliott V, Fernandez M, O'Neal L, et al. The REDCap consortium: building an international community of software platform partners. J Biomed Inform. 2019;95:103208.

71. Blom JW, Muth C, Glasziou P, McCormack JP, Perera R, Poortvliet RKE, et al. Describing deprescribing trials better: an elaboration of the CONSORT statement. J Clin Epidemiol. 2020;127:87-95. https://doi.org/10.1016/j. jclinepi.2020.07.011.

72. Thabane L, Akhtar-Danesh N. Guidelines for reporting descriptive statistics in health research. Nurse Res. 2008;15(2):72-81. https://doi.org/10.7748/nr2008. 01.15.2.72.c6331

73. Little RAA, Rubin DB. Statistical analysis with missing data. John Wiley \& Sons, Inc: Hoboken, New Jersey; 2002. https://doi.org/10.1002/9781119013563.

74. Ali M, Sherafali D, Fitzpatrick-Lewis D, Kenny M, Lamarche L, Mangin D, et al. Interventions to address polypharmacy in older adults living with multimorbidity: A review of reviews. Can Fam Physician. 2021. Accepted. .

75. Page AT, Clifford RM, Potter K, Schwartz D, Etherton-Beer CD. The feasibility and effect of deprescribing in older adults on mortality and health: a systematic review and meta-analysis. Br J Clin Pharmacol. 2016;82(3):583623. https://doi.org/10.1111/bcp.12975.

76. Braun V, Clarke V. Using thematic analysis in psychology. Qualitative Research in Psychology. 2006;3(2):77-101. https://doi.org/10.1191/14 78088706qp0630a.

77. Dedoose. 8.0.35 ed. Los Angeles: SocioCultural Research Consultants; 2018. p. Web application for managing, analyzing, and presenting qualitative and mixed method research data.

78. Lincoln YS, Guba EG. But is it rigorous? Trustworthiness and authenticity in naturalistic evaluation. New Directions for Program Evaluation. 1986; 1986(30):73-84. https://doi.org/10.1002/ev.1427.

79. Schwandt TA, Lincoln YS, Guba EG. Judging interpretations: But is it rigorous? trustworthiness and authenticity in naturalistic evaluation. New Directions for Evaluation. 2007;2007(114):11-25. https://doi.org/10.1002/ ev.223.

80. Mangin D, Dowson C, Mulder R, Wells E, Toop L, Dowel T, et al. The effectiveness of maintenance SSRI treatment in primary care depression to prevent recurrence: multicentre double blinded placebo controlled RCT. North American Primary Care Research Group Annual Meeting; Cancun, Mexico. 2015

\section{Publisher's Note}

Springer Nature remains neutral with regard to jurisdictional claims in published maps and institutional affiliations. 